\title{
Urgences
}

\section{Le passage : à la note}

\section{André Gervais}

Numéro 31, mars 1991

Poétique de la note

URI : https://id.erudit.org/iderudit/025638ar

DOI : https://doi.org/10.7202/025638ar

Aller au sommaire du numéro

Éditeur(s)

Urgences

ISSN

0226-9554 (imprimé)

1927-3924 (numérique)

Découvrir la revue

Citer cet article

Gervais, A. (1991). Le passage : à la note. Urgences, (31), 85-100.

https://doi.org/10.7202/025638ar

Ce document est protégé par la loi sur le droit d'auteur. L'utilisation des services d'Érudit (y compris la reproduction) est assujettie à sa politique d'utilisation que vous pouvez consulter en ligne.

https://apropos.erudit.org/fr/usagers/politique-dutilisation/
Cet article est diffusé et préservé par Érudit.

Érudit est un consortium interuniversitaire sans but lucratif composé de l’Université de Montréal, l'Université Laval et l'Université du Québec à Montréal. Il a pour mission la promotion et la valorisation de la recherche. https://www.erudit.org/fr/ 


\section{Le passage: à la note André Gervais}

Selon une première acception, il s'agit de mettre au bas de telle page ou à la fin de l'ensemble des pages le ou les éléments susceptibles d'appuyer, de compléter ou d'élargir ce qui figure sur lesdites pages, ce qui figure sur lesdites pages étant ici ce qu'il est convenu d'appeler le texte. Il s'agit de mettre en note (ce qui se rapporte au texte), d'ajouter une note (nécessaire à l'intelligence du texte). Selon une seconde acception, il ne s'agit plus d'annoter (un texte), mais bien de noter, de faire une note qui devient un (le) texte.

Dans l'annotation, le texte commande la note qui, bien sûr, peut être conduite de manière telle qu'elle rejoue le texte. Dans la notation, la note, subordonnée à aucun autre texte, s'autonomise en se textualisant. C'est le cas des notes, écrites depuis le début des années 1910 par Marcel Duchamp, et publiées à partir de 1914.

Trois notes de Marcel Duchamp, donc, dans trois domaines différents et à trois époques différentes. La première, dans le domaine des ready-mades, est un ordre donné à soi-même à l'infinitif, ordre finalement jamais exécuté dans l'ordre des choses plastiques (vers 1915-1916 / publiée en 1934 dans la Boîte Verte); la deuxième, dans le domaine des aphorismes, est aussi une façon de désigner un référentiel état de fait (années 1937-1945/publiée en 1980 dans Marcel Duchamp, Notes); la troisième, dans le domaine des signatures, est aussi, à l'impératif, ce qu'on appelle une note de service (1959/publiée en 1972 sous forme de carte postale) ${ }^{1}$.

1 La Boite Verte, « différentielle principale" du Grand Verre qui est, lui, la "trace majeure d'un processus" commencé, en fait, en 1912 et inachevé en 1923 (selon les formules de Michel Butor: "Reproduction interdite", Critique, Paris, no 334, mars 1975, p. 276), est le second recueil de notes (écrites pour une très grande part entre 1912 et 1915) publié par l'(an)artiste. Marcel Duchamp, Notes : ce titre - qui est de l'éditeur (Paul Matisse) et/ou du préfacier (Pontus Hulten) - annexe littéralement le nom de l'auteur tout en se séparant de lui le temps et l'espace d'une virgule, celle-là même qui, dans le titre du Grand Verre (La Mariée mise à nu par ses célibataires, même), ne sera "formellement incorporée" qu'en 1936 (selon la formule de Jean Suquet: Le guéridon et la virgule, Paris, Bourgois, 1976, p. 84). Enfin, pour ce qui est de la carte postale, voir note 11 . 


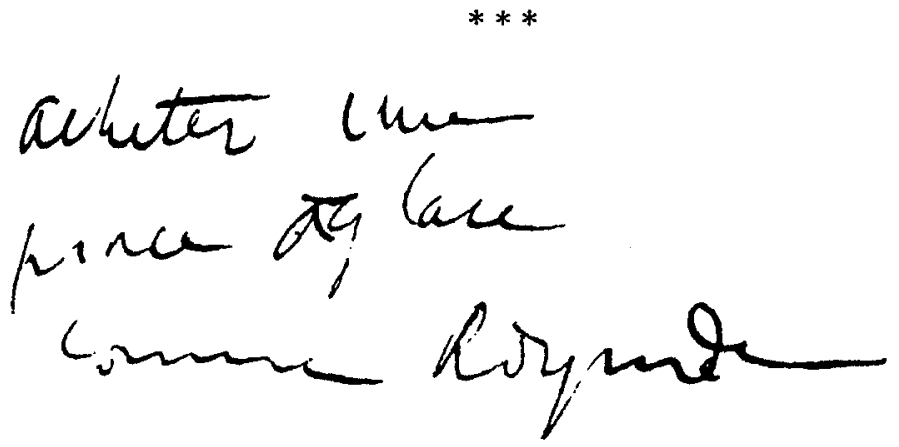

Duchamp a choisi des ready-mades sans que ceux-ci soient accompagnés d'une note, annoncés ou commentés par celle-ci; inversement, des notes à propos de ready-mades sont restées, plastiquement, lettre morte. En voici une, manifestement griffonnée.

Le ready-made est un objet d'art paradoxal, entre la peinture et l'écriture, une œuvre délibérément ouverte. Cet objetci, resté à l'état de projet, est une phrase minimale qui va d'un verbe (au degré zéro de la conjugaison) à un nom (à la généricité étrange). Ce qui n'empêche pas, naturellement, d'inscrire cette phrase dans l'ensemble textuel et de la faire résonner.

Pour la pelle à neige et la pince à glace, deux noms à charnière, le corps est là: la pelle à neige n'est-elle la métaphore et la métonymie du bras (In Advance of the Broken $A r m$, novembre 1915 , premier ready-made à coïncider avec le nom générique de ready-made), la pince à glace des bras ( two arms joined at one end by a pivot or hinge ${ }^{2}$ ), des mâchoires ("deux mâchoires à levier, articulées entre le point d'appui et le point d'application de l'effort ${ }^{3}$ ), voire de la langue (mother tongue)? Il n'en faut pas plus pour que cette pince à glace, par le détour de l'autre langue (ice-tongs/his tongue), ne manipule plus des blocs de glace, mais désigne plutôt tel intertexte. L'épisode, célèbre en effet, des "parolles gelées " 4 , qui est un discours sur l'interprétation, met en

\footnotetext{
2 The American Dictionary of the English Language.

3 Larousse du XX siècle.

4 François Rabelais, Le Quart Livre, chapitres LV et LVI, dans CEuvres complètes, tome II, édition de Pierre Jourda, Paris, Garnier, 1962, p. 203-208.
} 
scène Pantagruel pour qui les paroles ne peuvent que dégeler, et ses compagnons pour qui les paroles, déjà réifiées, "sembloient dragée perlée de diverses couleurs »:

Pantagruel dénonce sans ambiguïté l'absurdité des paroles gelées: le langage ne se conserve pas, mais se recrée constamment, au gré de l'émetteur et du récepteur. [...] Du côté du gel et de la violence, Rabelais semble donc ranger l'écrit, associé à la primauté de la vision, en contraste avec l'oral et l'ouïe, dont Pantagruel semble revendiquer la supériorité. La rigoureuse binarité du thème se précise: camarades - gel - perceptions sensuelles - significations univoques - voir - écrit - littéraire / Pantagruel - dégel perception étagée - significations plurielles - écouter oral-vécu 5 .

En ce sens, rien de plus utile qu'une pince à glace, objet univoque, pour des significations univoques, déterminées: telles paroles, par exemple, " sont, par Dieu ! coups de canon " et, dans le contexte socio-politique européen entre 1914 et 1918 , il y a bien telle "grosse et felonne bataille ". Mais ici ce n'est plus le "feurre bien nect" (la paille bien nette) - ou le bran de scie - et la glacière, c'est le glossaire des paroles " assez mal plaisantes à veoir " qui est monnayé en exemples: "his» (tiens, on dirait un mot anglais), «lorgne " (ne s'agit-il pas, métatextuellement, de " veoir"), "trr " (tiens, on dirait le " tr" si important dans Jeune homme triste dans un train (1911) et dans Trébuchet (1917), par exemple), "magoth " (ne s'agit-il pas, illocutoirement, d' ' acheter "), entre autres. Cette "pince àg lace", comme le propose la graphie manuscrite, n'at-elle pas elle-même partie liée avec le glossaire, ainsi que ce "Rdyme" (5 lettres comme "pince" et comme "glace»), dont la dernière lettre est surchargée d'un "de " (où " au sujet de " se lie au sujet «M.D. »)?

Mais une pince à glace, par une espèce d'ironie à froid, ne met-elle pas rigoureusement en équilibre, comme le corps et l'esprit chez Rabelais, les deux locutions "être chaud de la pince " et «être de glace»?

****

5 Michel Jeanneret, «Les paroles dégelées ", Littérature, Paris, $n^{\circ} 17$, février 1975, p. 20. 
88

* Gruyère plombé pour dentitions défectueuses *

Sur le modèle $\mathrm{X}(+\mathrm{A})$ pour $\mathrm{Y}(+\mathrm{B})$, Duchamp met directement en relation tel "fromage" et tel "dommage", pour reprendre deux des termes d'un aphorisme calligrammatique publié en 1958. N'y a-t-il pas, tout simplement, quelque rapport entre un fromage à pâte (pressée et cuite) et de la pâte à dents? Cette jointure: fromage à/pâte/à dents, d'où, rousselliennement, fromage à dents. Au premier les (grands) trous, aux secondes les (petites) cavités. Ne s'agit-il pas de plomber un fromage comme on plombe des dents, de l'obturer avec un alliage? X pour Y, c'est, infratextuellement, "œil pour œil, dent pour dent", les yeux de ce fromage pour les cavités de ces dents, et c'est, métatextuellement, la loi du talion pour cet alliage. Non seulement y a-t-il croisement (œil pour dent), mais marquage de ce croisement par une rime (talion / alliage).

Vers une dentition sans dents, une dentition molle comme il y a le ready-made mou (...Pliant... de voyage, 1916), housse de machine à écrire Underwood sans machine à écrire ${ }^{6}$. Une fois le fromage dissous, restent, bien sûr, les plombages, ces drôles de cailloux que l'art dentaire propose à tous les Démosthène de la tentative orale (à moins qu'ils aient déjà du plomb dans l'estomac, à défaut d'en avoir dans la tête). De même, une fois l'écriture impersonnelle réalisée, restent les idées reçues, ces phrases avec lesquelles on parle sans parler:

Je crois que l'ensemble serait formidable comme plomb. II faudrait que, dans tout le cours du livre, il n'y eût pas un mot de mon cru, et qu'une fois qu'on l'aurait lu on n'osât plus parler, de peur de dire naturellement une des phrases qui s'y trouvent ${ }^{7}$.

Le cours de ce livre (comme d'un fleuve), chez Flaubert, fait advenir le plomb pour la ligne d'écriture, les caractères d'imprimerie, puis, par polysémie, le plomb pour la ligne de pêche - mais ici, on le voit, c'est plutôt pour l'empêchement. Chez Duchamp, autre aphorisme: "Le plomb est le premier métal ", façon de dire, en fondant (fondre/fonder) le

6 Ici le jeu over (dust cover)/ under (Underwood) et, naturellement, le désir de trousser (tiens, encore le " $\mathrm{tr}$ ") la dactylo.

7 Gustave Flaubert à Louise Colet, 16 décembre 1852, à propos, déjà, du Dictionnaire des idées reçues; dans Correspondance, tome II (1851-1858), édition de Jean Bruneau, coll. "Bibliothèque de la Pléiad e", Paris, Gallimard, 1980, p. 208-209. Cette lettre est publiée dès 1910, au moins, dans l'éd. Conard. 
métatextuel et le textuel, que lire sur la composition même est le premier métalangage. Façon paradoxale de dire, bien sûr, pour celui qui a publié ses notes en ne les faisant pas composer, en les laissant dans leur scription et sur leur support ${ }^{8}$. Façon, justement, de tomber à plomb.

Entre la poire et le fromage, surtout lorsque la poire est cet objet à l'extrémité d'un fil qui sert de déclencheur dans un appareil photo, ou encore, par polysémie, lorsque la poire est un visage. Sur la couverture du catalogue de l'exposition First Papers of Surrealism (New York, octobre-novembre 1942), deux photos: une tranche de gruyère (p. 4), un mur de grange (p. 1). Le fromage est suisse et Kurt Seligmann, qui possède la maison de campagne et la grange, l'est également. Sur la p. 1, cinq perforations correspondant aux cinq coups de fusil tirés par Duchamp sur ledit mur; sur la p. 4, le titre et les autres coordonnées. Dans ce catalogue, entre autres, les "Compensation Portraits» des surréalistes, par lesquels la dette des hommages est détournée en images, Duchamp cadrant le sien à partir de la photo d'une femme par Ben Shahn (compensation/Ben, Duchamp/Shahn) ${ }^{9}$. Obturer (les trous d')un fromage peut alors s'inverser en perforer (la photo d')un mur de grange: rendre la pareille c'est prendre l'appareil (œil/ obturateur), et exposer tels " first papers" c'est, déjà, composer avec le " premier métal ".

"Gruyère plombé... » est rajouté à la mine de plomb sur le morceau de papier qui contient au recto et au verso telle note (N 26) écrite à l'encre noire et corrigée à la mine, relative à la catégorie de l'«infra-mince». Il ne peut donc être dans la section "Jeux de mots" (N 208-289) qui contient la grande majorité des aphorismes. Il y a dans cette note, toutefois, ceci qui peut être utile: "fondage (dans les liquides avec le sucre pax.)». Seul «l'état actif et non pas le résultat» de cette

8 De son vivant, Duchamp n'a publié que deux livres au sens ordinaire du mot: un traité d'échecs, écrit en collaboration avec Vitaly Halberstadt (L'opposition et les cases conjuguées sont réconciliées, Paris/Bruxelles, Ed. de l'Échiquier, 1932), et un recueil d'aphorismes (Rrose Sélavy, Paris, Guy LévisMano, 1939).

9 En compensation/en revanche (synonymiquement)/vers Ben Shahn (par anagramme phonique). Ce nom n'est pas sans également rappeler, par son initiale, le $S$ de Sélavy ainsi que, par ses 5 lettres, les 5 lettres de Rrose. Le passage de -champ à Shahn est bien celui du $c$ masculin au $S$ féminin. Voir André Gervais, La raie alitée d'effets. Apropos of Marcel Duchamp, coll. "Brèches ", Montréal, HMH, 1984, p. 25 et suiv. Enfin, de Seligmann à Shahn comme des 5 coups auxdites 5 lettres. 
expérience - la note contient une quinzaine de noms en -age d' "expériences" possibles - doit être gardé. Le salé de la tranche de fromage (p. 4) peut aussi s'inverser en le sucré du mur de la grange (p. 1), surtout lorsqu'on constate que Kurt Seligmann habite, dans l'État de New York, à... Sugar Loaf. Entre les pages de la couverture du catalogue, la distance active est remarquablement courte.

Autant cet aphorisme désigne aussi le fait que Duchamp a une mauvaise dentition 10 , autant tel ready-made ( $N o$ Smoking, 1950) rappelle a contrario sa propension à fumer.

\section{DON'T FORGET}

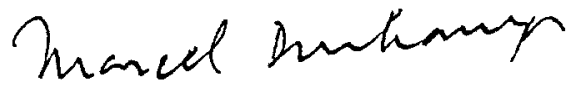

10 En témoignerait cette anecdote (des années 1940, selon toute vraisemblance), rapportée par Alice Goldfarb Marquis dans sa biographie (Marcel Duchamp: Eros, c'est la vie, Troy, The Whitston Publ. Co., 1981, p. 281): "David Mann raconte que Duchamp était si pauvre qu'il lui avait fallu se rendre pour un traitement dentaire jusqu'à une clinique de Brooklyn parce que les soins y étaient gratuits. Le dentiste lui avait arraché tout un groupe de dents, et en 
Je laisse à Richard Hamilton le soin de présenter les circonstances ${ }^{11}$ :

J'avais besoin d'une signature afin de la reproduire dans l'édition en livre de la Boîte Verte à laquelle j'étais à mettre la dernière main. II me l'a envoyée sur un morceau de papier avec "DON'T FORGET" comme en-tête; peut-être avezvous vu cela, nous en avons fait une carte postale récemment, Hansjorg Mayer et moi. Nous avons pris soin de ne pas dire sur la carte postale que ceci est une œurre d'art de Duchamp. Maintenant, je suis tout à fait sûr que le placement [placing] de cette signature sur la petite feuille [slip] est une idée superbe autant que mûrement réfléchie. II aurait pu utiliser n'importe quel morceau de papier mais le bloc [pad] aura sans doute été la chose utile le plus à la portée de sa main. Cela m'apparaît tout à fait comme une image duchampienne typique.

Cet envoi date de 1959 , probablement après septembre puisqu'à cette date Duchamp est à Londres pour la suite d'un entretien avec Richard Hamilton, justement, artiste pop anglais et éditeur d'une "version typographique" (par luimême) de la première traduction complète en anglais (par George Heard Hamilton) des notes de la Boîte Verte. Le livre ${ }^{12}$ contient deux préfaces précédées de cette note pour laquelle il fallait ladite signature:

This version of the Green Box is as accurate a translation of the meaning and form of the original notes as supervision by the author can make it.
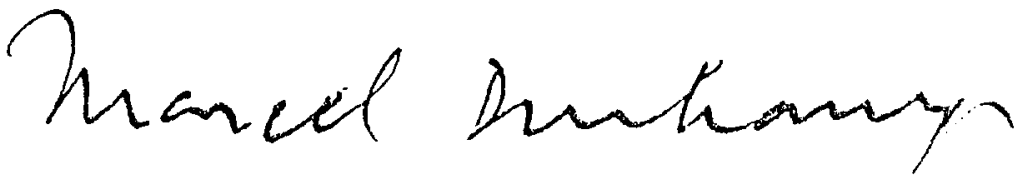

New York 1960

revenant Duchamp avait eu un grave malaise sur le bord d'un trottoir; il s'était reposé un peu puis était rentré chez lui. " (Ma trad.)

11 Richard Hamilton, "Letter to Alison Knowles " [fin 1972 ou début 1973], dans Collected Words. 1953-1982, Londres, Thames and Hudson, [1982], p. 236. (Ma trad.) Les p. 181-238 portent sur l'œuvre duchampienne.

12 Si la boîte est, en effet, devenue un livre, et les dessins et plans ont été refaits (et "embellis " comme le sera la copie du Grand Verre, en 1965-1966, par 
92

Hamilton fait bien les choses en appelant cela un "reminder», c'est-à-dire un pense-bête (et l'on ne peut pas ne pas entendre tel sic dans la dernière phrase d'un entretien de 1946: "I am sick of the expression "bête comme un peintre" "), un mémento, cette note destinée à rappeler ce que l'on a projeté de faire. Or, il ne s'agit plus de faire, mais d'être (Marcel Duchamp). Dans le même entretien, à propos de son travail de l'année 1912, particulièrement, il précise: "I was interested in ideas - not merely in visual products. I wanted to put painting once again at the service of the mind. " $\mathrm{Ce}$ "reminder" sert à rappeler cela aussi. D'ailleurs, le " re " (referring to) encadrant "mind» peut être tiré des deux mots de 2x3 lettres que sont «FORGET" et «Marcel», où $r$ et $e$ sont, dans les deux cas, les $3^{\text {e }}$ et $5^{e}$ lettres ${ }^{13}$. Ce "re-"/ «-er" fait mieux voir la griffe des première et dernière lettres (vers la gauche/vers la droite) de ladite signature ${ }^{14}$. On le voit déjà, la connivence entre les deux lignes (l'une imprimée en majuscules, impersonnelle, l'autre manuscrite, alternant majuscules et minuscules comme il se doit) est grande. Mais il y a aussi : horizontalement (le $T$ par lequel se terminent "DON'T» et "FORGET») et en diagonale (le $D$ par lequel commencent "DON'T" et "Duchamp", le $M$ de "Marcel" renvoyant, parce qu'il en devient le gond, à l'un ou à l'autre).

Mais qu'est-ce que cela peut bien signifier, en cette époque où la reconnaissance internationale de l'œuvre et de la personne n'a jamais été aussi grande? Au lieu de noter un message en vue de (du genre "n'oublie pas d'envoyer à Richard Hamilton une signature"), Duchamp fait de la petite feuille d'un bloc (slip of a pad) le lieu même d'un glissement (slip) par lequel une note de service en vient à être «at the service of the mind», devient une signature: sa signature. Cet

le même Hamilton), il faut dire que la mise en page avec choix de couleurs d'encre est plutôt réussie en tant qu'essai pour rendre la pareille. Le livre est paru à Londres, chez Percy Lund, Humphries \& Co. Ltd., et à New York, chez Wittenborn, en 1960.

133 et 5 sont, depuis les années 1910, deux des chiffres organisateurs de la textualité duchampienne: voir La raie..., p. 328. "Ben Shahn ", c'est aussi 3 et 5 lettres.

14 Faut-il rappeler cette note: "Reading between the lines/Riding between the lions - " (N 261) où la reconnaissance de l'infratexte - texte inscrit entre les lignes d'un autre texte - est liée au danger de l'expédition lectorale. Voir La raie..., p. 288. II va sans dire que, si la part du lion est celle du texte, la griffe est aussi celle du lecteur. 
agencement, qui est d'ordre privé (from a pad to a chum, par anagramme graphique ${ }^{15}$ ) et en vue de (fournir une signature qui sera imprimée dans un livre), ne sera connu que plus tard, si le destinataire l'a conservé, et deviendra utile si se posent avec une certaine acuité les questions de l'artiste et de l'anartiste, du majeur et du mineur, de l'œuvre considérée telle et des autres gestes, aussi divers et intimes soient-ils, qui l'entourent, la relancent, la désignent.

Un autre geste de la même année permet cependant d'apporter quelques précisions. À l'occasion de la publication de Sur Marcel Duchamp de Robert Lebel, simultanément en français et en anglais, une petite exposition, sans affiche mais en présence de, est organisée à la Sidney Janis Gallery à New York (6 avril-2 mai), puis, en mai (à partir du 5), avec affiche mais en l'absence de, à la librairie La Hune à Paris. Le 5 mai 1959, donc, Marcel Duchamp, alors à Cadaquès, envoie, pour l'ouverture, le télégramme suivant: “JE FAIS SOUS MOI» 16. L'affiche reprend l'Autoportrait de profil (1958), avec l'ajout du nom de l'(an)artiste ${ }^{17}$ en deux couleurs ( $M$ en rouge orangé, $A$ en bleu pâle, etc.) et des coordonnées nécessaires. L'autoportrait est fait par soustraction, selon le tracé d'une matrice métallique, dans le coin supérieur gauche d'un carré, bleu pâle lui aussi. Le profil ainsi obtenu, tout en participant du fond noir de l'affiche, regarde, pour ainsi dire, sa signature — «marcel dechiravit» - dans le coin inférieur droit dudit carré: l'un (est) fait, l'autre dit qui a fait et ce qui est fait. Le rapport complexe entre les instances sur l'affiche n'est pas sans annoncer le rapport entre les pronoms dans le télégramme.

Mais tout ceci ne désigne-t-il pas surtout tel intertexte: "Rapsodie du sourd" de Corbière ${ }^{18}$, où l'on retrouve justement

15 Ce «chum" (ou « chap ", puisqu'il est ici interpelé, en tant que destinataire, par le “DON'T FORGET ") est bien l'" ami » Hamilton: de Duchamp (et * slip ") à Hamitton, donc.

16 Cité par Ecke Bonk, Marcel Duchamp The Box in a Valise. Inventory of an Edition, New York, Rizzoli, 1989, p. 183.

17 C'est en septembre 1959, justement dans la seconde partie de cette interview par Richard Hamilton, que Duchamp proposera, pour échapper à l'opposition artist/anti-artist, de forger le mot anartist, "meaning no artist at all $"$ : « I don't mind being an anartist. $"$

18 Tristan Corbière, Les amours jaunes (1873). Le poème est dans la section intitulée * Raccrocs". Faut-il rappeler qu'une hune est une plate-forme demicirculaire à l'avant d'un voilier, ce qui nous renvoie à la section intitulée «Gens de mer " du mème recueil. Les deux sections, si on les numérote en chiffres 
94

un je t'ai à l'œil: «À l'œil - Mais gare à l'œil jaloux, gardant la place/De l'oreille au clou !...» (l'œil remplaçant l'oreille pour le sourd), un "Je suis là, mais absent..." (absent de Paris pour l'ouverture de l'exposition, absent aussi, en creux sur l'affiche, par effet d'obscurcissement), et surtout ce $*-$ Rien - Je parle sous moi...» qui n'hésite pas à faire entendre que «sourd» est fait, duchampiennement, de "sous" et de la finale "-rd"! Je continue à faire - l'art, c'est faire, et faire, c'est choisir ${ }^{19}-$, malgré tout ce que les regardeurs considèrent comme étant de moi : Lebel a fait Sur..., eh bien, je fais sous... Il y a l'œuvre overground, bien sûr, il y a aussi le travail underground (Étant donnés...) qui ne sera exposé que posthumément. Il y a encore, peut-être ce mois même, cette "tête comme un bon cercueil" dûment intitulée Sculpture-morte 20 . Mais il y a plus. «Toujours, comme un rebus, je travaille à surprendre/Un mot de travers... - Non - On m'a donc oublié !» Non seulement «marcel», en se déchirant vite, est-il comme un rebut, un déchet ravi, tout en étant, autre possible latin, un rébus, une énigme ${ }^{21}$, non seulement inverse-t-il deux fois les lettres de l'«art", mais il lit un «donc oublié » qui, hors ce rapport texte/ intertexte, conduit à "DON'T FORGET»! L'angrais, qui traduit au signifié (oublier/forget) et au signifiant (donc/ don't), $y$ est pour quelque chose.

La catégorie de l'«infra-mince» - Denis de Rougemont le note en 1945 dans son journal - a occupé Duchamp pendant

romains - voir la signature clatine" de l'autoportrait -, sont l'inverse l'une de l'autre (V/VI) tout comme " Lebel " est un nom palindromique !

19 Ce que Duchamp rappellera à Georges Charbonnier dans ses entretiens (décembre 1960-janvier 1961). Je renvoie, pour les citations, à Thierry de Duve, Nominalisme pictural, coll. « Critique «, Paris, Minuit, 1984, p. 247 (voir la section, p. 246-250).

20 Ce jeu sur le patronyme - le corps comme bière - se retrouve aussi dans Torture-morte. Ces deux œuvres, et une troisième, sont d'ailleurs faites à Cadaquès où il séjourne alors (entre la mi-avril et la fin juin), pour un autre livre de Robert Lebel que ce dernier ne commencera même pas. Voir La raie..., p. 336, n. 1.

21 Sur cette question de l'énigme, suggérée ici par le mot-valise de Corbière, je propose, pour éviter toute ambiguilté, de reconduire ce que disent Harry Mathews et Georges Perec à propos de Roussel (" Roussel et Venise. Esquisse d'une géographie mélancolique ", L'Arc, Aix-en-Provence, $n^{\circ} 68,1 \propto$ trim. 1977, p. 21): «il n'y a pas de mystère Roussel, son cuvre ne constitue pas une énigme à résoudre; c'est notre lecture seule, notre soif d'explications, notre goût pour les tenants et les aboutissants, qui suscite autour d'elle cette impression d'un secret à forcer. [...] Toute explication de Roussel achoppe en fin de compte sur la seule évidence d'une méthode infinie." 
les dix années précédentes ou à peu près ${ }^{22}$. Mais ce n'est qu'en 1980, dans les Notes posthumes, que les fragments relatifs à cette catégorie (N 1-46) sont disponibles.

Pas de définition comme telle - de la même manière, Duchamp ne définira pas l'art dans Le processus créatif -, mais une graphie flottante (inframince, infra-mince, infra mince) et un certain nombre d'exemples, associés aux 5 sens. Quelques-uns:

a) infra-mince visible: «Peinture sur verre vue du côté non peint donne un infra mince." (N 15);

b) infra-mince tactile: "Papier creux (intervalle inframince sans qu'il y ait pour cela 2 feuilles)" (N 17);

c) infra-mince auditif: «Pantalons de velours - leur sifflotement (dans la marche) par frottement des 2 jambes est une séparation infra mince signalée par le son." (N 9);

entre a) et b): "Loupe pour "toucher" - inframince" (N 11);

entre a) et c): «Séparation infra mince entre le bruit de détonation d'un fusil (très proche) et l'apparition de la marque de la balle sur la cible - (distance maximum 3 à 4 mètres. - Tir de foire)" (N 12);

d) infra-mince olfactif (et gustatif): "Quand la fumée de tabac sent aussi de la bouche qui l'exhale, les 2 odeurs s'épousent par infra mince" (N 11 - le seul texte connu du vivant de Duchamp, publié en 1945).

La «société anonyme des porteurs d'ombre représentée par toutes les sources de lumière (soleil, lune, étoiles, bougies, feu -)" (N 3) fait de tous ces corps, que ce soit le jour ou la nuit, des "habitants de l'infra-mince" (N 34) - comme si l'infra-mince était une ville - qui, bien qu'ils «travaillent dans l'infra mince» (N 3) sont bien, par oxymore, des "fainéants" (N 34) ou, mieux encore, des "Extatiques esthétiques" (N 41). Cette façon de faire en n'ayant rien à faire est le travail de celui qui fait « des œuvres qui ne soient pas d" art" "(dit une note de 1913), le travail de l'artiste inerte: en un mot, de l'anartiste, du désœuvré ou flemmard, anagramme de «flamme» (voir "bougies, feu») et, ici encore, 
96

de la finale «-rd»! L'extase, étymologiquement l'kaction d'être hors de soi», est soit mystique, soit pathologique; ici détournée, elle devient esthétique. C'est par S., l'alter ego, que vient la paresse et c'est dans Rrose Sélavy que le ravi se trouve distendu: de "One oiseau oisif cherche noise en Seineet-Oise sur la paroi parée de paresse de paroisse», aphorisme des années 1930 allongé dans les années 1960, à «La querelle de l'ombre portée dans son rapport avec l'infra-mince " (N 40), rien ne se dit qui n'ait déjà été mis en place ${ }^{23}$. L'ombre portée l'est, bien sûr, par les porteurs d'ombre, comme l'eau par les porteurs d'eau.

Ce n'est pas la seule fois que Duchamp détourne la terminologie religieuse. Dans Le processus créatif (brève allocution, 1957), n'écrit-il pas que «avec le changement de la matière inerte en ouvre d'art, une véritable transsubstantiation a lieu et le rôle important du spectateur est de déterminer le poids de l'œuvre sur la bascule esthétique "; dans L'artiste doit-il aller à l'université? (autre brève allocution, 1960), n'ajoute-t-il pas que "aujourd'hui plus que jamais l'Artiste a cette mission para-religieuse à remplir: maintenir allumée la flamme d'une vision intérieure dont l'œuvre d'art semble être la traduction la plus fidèle pour le profane». Tout ceci doit être entendu sur fond d'évacuation générale: «en termes de "métaphysique populaire" je n'accepte pas de discuter sur l'existence de Dieu - ce qui veut dire que le terme "athée" (en opposition au mot "croyant") ne m'intéresse même pas " écrit-il (4 octobre 1954) à André Breton ${ }^{24}$; ou de recherche indéfinie du pat: "Toutes ces balivernes, existence de Dieu, athéisme, déterminisme, libre arbitre, sociétés, mort, etc., sont les pièces d'un jeu d'échecs appelé langage et ne sont amusantes que si on ne se préoccupe pas de "gagner ou de perdre cette partie d'échecs" "écrit-il (8 mars 1956) à Jehan Mayoux.

La «séparation infra-mince», à la fois «intervalle" et «cloison» (N 9), pour arriver à l"«osmose esthétique», principe

23 La querelle, ici, s'inscrit dans le paradigme de la bagarre (La bagarre d'Austerlitz, ready-made, 1921), des démêlés (" Daily lady cherche démélés avec Daily Mail.", aphorisme publié sous cette forme allongée en 1939) et de l'opposition (L'opposition et les cases conjuguées... : voir note 8), entre bien d'autres mises en situation conflictuelles.

24 Dans les Notes, on trouve un "St Sulpice de Tantale " (N 237) où sont ainsi fondus, plutôt qu'opposés, un saint chrétien et un héros païen. 
du processus créatif (entre l'artiste et le regardeur via l'œuvre), se lit dans le devenir-peau du "possible" - "La possibilité de plusieurs tubes de couleur de devenir un Seurat est l' $l^{\text {' }}$ explication" concrète du possible comme infra mince" (N 1) -, par lequel se met en place la "réciprocité " (N 3) et où se produit l'«échange" (N 10). Ce devenir-peau fait de la séparation une séparation diaphane, pas opaque, donc, bien que quelquefois transparente (voir N 32). Le corps du porteur d'ombre est là, désigné d'infra-mince par la lumière, et qu'il s'agisse du «pantalon porté et très marqué de plis " auquel il s'agit d'" adapter l'infra mince gorge de pigeon * (N 44) ou du "pastel de pellicules tombées des cheveux» (N 20) sur le "papier creux" déjà cité, tout infra-mince d'infra-mince, même, passe par lui. Ainsi "guest" et "host", regardeur et artiste en l'occurrence, peuvent-ils être fondus en "ghost», ce "Fantôme qu'à ce lieu son pur éclat assigne ", dans telle équation proposée justement à l'occasion d'un vernissage en $1953{ }^{25}$.

"Le système métrite par un temps blennhoragieux": cet aphorisme publié en 1924 incorpore, en les érotisant, l'intervalle (le temps mâle) et la cloison (l'espace femelle), sans oublier la forme en S, future initiale de Sélavy, du * mètre diminué" - les initiales, ici, sont déjà celles de Marcel Duchamp — dans les 3 stoppages étalon (1913-1914). En effet, intervalle: " en essayant de mettre 1 surface plane à fleur d'une autre surface plane on passe par des moments infra minces" ( $\mathbf{N} 45)$; et cloison: «Reflets dépolis donnant un effet de réflexion - miroir en profondeur - pourraient servir d'illustration optique à l'idée de l'infra mince comme "conducteur" de la $2^{\mathrm{e}}$ à la $3^{\mathrm{e}}$ dimension" (N 46). Mais que dire de cette spéculation (lettre à Serge Stauffer, 21 mai 1961): «bien entendu aucun de nos sens ne trouve d'application quadridimensionnelle sauf peut-être le toucher ["Caresses infra minces" (N 28)] et par conséquent l'acte d'amour comme sublimation tactile pourrait faire entrevoir ou plutôt entretoucher une interprétation physique de la 4me dimension " ${ }^{26}$.

25 Le vers de Mallarmé, tiré du sonnet dit du cygne, désigne cet oiseau, blanc dans le blanc (de la neige et de la glace), qu'il est possible de reconnaître encore dans l'“oiseau oisif" de l'aphorisme déjà cité. Voir La raie..., p. 196. " A Guest + a Host $=$ a Ghost" ou, si l'on veut, un hóte + un hôte = un autre (tiens, toujours le $* \operatorname{tr} ")$.

26 L'aphorisme valéryien (dans L'idée fixe, Paris, Gallimard, 1934) n'est pas loin: "Ce qu'il y a de plus profond dans l'homme, c'est la peau". A fleur de (affleurer) est fleur de (effleurer). 
98

On le constate, l'infra-mince est bien un nom (comme "extatiques", adjectif ici devenu nom) et un adjectif (comme " esthétiques ", nom ici devenu adjectif), bien qu'il soit précisé, en vain semble-t-il, de "ne jamais en faire un substantif * (N 5). Et «extatiques», n'est-ce pas, en anglais, "ecstatics", nom qui emprunte à deux néologismes - "estastic estactic" (N 273) - , deux adjectifs ne différant que par un s/c, ainsi Marcel de Sélavy, qui sont en fait la condensation de deux propositions inverses faites d'ailleurs de noms et d'adjectifs, l'une dysphorique (estastic: fantastic esthetics and taste), l'autre euphorique (estactic: tact and tactical esthetics) ${ }^{27}$.

Le travail du fainéant est bien celui qu'impliquent aux deux bouts de l'œuvre d'une part, depuis les années 1910 , le ready-made, " a work of art without an artist to make it » ditil en 1963 à Francis Roberts, d'autre part Étant donnés..., pendant vingt ans (1946-1966) underground, comme il a été dit, alors qu'overground, apparemment, l'inactivité artistique s'est installée. La paresse du fainéant est bien ici, étymologiquement, la feinte de son travail 28 , quand l'extase du même n'est peut-être que le nom, selon Mallarmé, de sa "disparition élocutoire». La paresse du fainéant: «Ce que je suis? Est-ce que je sais? Un homme, tout simplement, un "respirateur" " dit-il en 1954 à Michel Sanouillet; "Je ne considère pas que le travail que j'ai fait puisse avoir une importance quelconque au point de vue social dans l'avenir. Donc, si vous voulez, mon art serait de vivre; chaque seconde, chaque respiration est une œuvre qui n'est inscrite nulle part, qui n'est ni visuelle ni cérébrale. C'est une sorte d'euphorie constante " dit-il en 1966 à Pierre Cabanne.

Et si tout ceci, élégamment, était une allégorie: «l'allégorie (en général) est une application de l'infra mince " (N 6). Comme chez Ponge, à partir des années 1920, chez qui les termes allégorie, application et diaphane sont aussi présents. Bernard Beugnot, dans une récente monographie consacrée à ce dernier, cerne bien cet aspect ${ }^{29}$ :

27 Dans les Notes, on trouve encore «Tact et tactique / Tact and tactics" (N 230), formule française et anglaise qui vient corroborer cette dernière proposition, la seule qui soit, en fait, duchampiennement recevable, opérante.

28 Fainéant: « De fais, et néant, altér. de faignant, ppr. de feindre, au sens anc. "paresser" "(Dictionnaire du français Plus).

29 Bernard Beugnot, Poétique de Francis Ponge. Le palais diaphane, coll. "Écrivains", Paris, PUF, 1990, p. 95-96. Voir *Au commencement était la fable ", p. 76-100. 
Le genre hérité de la tradition subit des transformations qui en rendent le visage moins reconnaissable: substitution partielle d'objets aux êtres animés, disparition de la partie narrative au profit de la description dont la temporalité n'est plus celle de l'anecdote, mais de la mémoire lexicale et sémantique ou de l'aventure organique, effacement par diffusion sur toute la surface du texte de la dichotomie récit/ morale, amuïssement de la règle de vie qui ou bien s'abolit totalement ou bien se fond dans une leçon d'ordre poétique. C'est dire que le rapport des deux plans se trouve du même coup modifié; plus de hiérarchie, de séparation entre le plaisir de conter et le souci didactique, mais une réciprocité où objet et poétique trouvent place, l'un par rapport à l'autre, dans un système circulaire.

Titres, inscriptions et objets que sont les ready-mades, notes descriptives publiées telles qu'elles furent écrites (griffonnées ou recopiées) relatives au Grand Verre ${ }^{30}$, complexes jeux de mots et fréquente métatextualisation des aphorismes, toutes ces caractéristiques connues de l'œuvre duchampienne sont recoupées, on le voit, par le passage que je viens de citer.

Ainsi en est-il également des paragraphes qui terminent l'introduction de la première lettre (15 mars 1952) envoyée à Marcel Jean qui lui avait posé 33 questions ${ }^{31}$ :

If est seulement curieux de constater combien la mémoire est fragile même pour les époques importantes de la vie -

C'est d'ailleurs ce qui explique la fantaisie heureuse de l'histoire.

L'«explication", jamais donnée, est le mot valant autant pour le possible de l'œuvre («un Seurat») que pour l'histoire de l'individu ("les époques importantes de la vie») qui a partie liée, désormais, avec l'histoire de l'art. L'«explication", indéfiniment réactivée, est le processus créatif ${ }^{32}$. Mais c'est

30 Voir André Gervais, " Duchamp et du pré, manuscrits", Urgences, n० 24 (n ${ }^{\circ}$ intitulé Le manuscrit sous l'angle génétique), juillet 1989, p. 53-65, pour une "comparaison" de la Boîte Verte de Duchamp et de La fabrique du pré de Ponge.

31 Marcel Duchamp, Lettres à Marcel Jean, Munich, Verlag Silke Schreiber, 1987, p. 42. Les vingt missives sont éditées et annotées en trois langues (allemand, français, anglais).

32 A cet effet, je propose, sur le modèle syntaxique de la N1 ("un Seurat"), de réécrire ainsi ces paragraphes: « La fragilité de la mémoire même pour les 
un autre mot qui revient dans l'épitaphe, écrite une douzaine d'années plus tard: “... et d'ailleurs c'est toujours les autres qui meurent" (N 252 et 256). En ses début et fin, après un "et " rétablissant indéfiniment le contact, * d'ailleurs * et "meurent ", au-delà de la rime, mettent en scène *I*, le sujet dit «M.D. *, dans ses œuvres, et c'est bien de ce * d'aill-» que les autres, parce que ça n'arrive qu'à eux, «meurent» ("die»).

Et si, à propos des notes de la Boîte Verte, le "reminder"

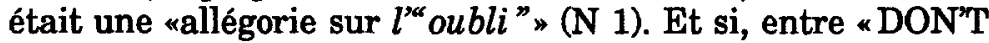
FORGET " et "chaque respiration est une œuvre qui n'est inscrite nulle part ", entre l'art et la vie ${ }^{33}$ ainsi articulés, il y avait une " analogie inframince" ( $\left.\mathrm{N}_{2}\right)$.

Quand Duchamp discute avec Michel Sanouillet, au dîner Rrose Sélavy (15 mai 1965), sur l'éventuel titre à donner à l'ensemble de ses écrits, il écarte la possibilité que celui-ci soit Prendre Duchamp ${ }^{34}$. Prendre et prétendre le posséder, comme on possède financièrement ou sexuellement, l'utiliser comme un outil, comme allant de soi, etc. Don't forget me, but don't try to get me.

époques importantes de la vie est l'cexplication " abstraite de la fantaisie heureuse de l'histoire."

33 La vie, aussi quotidienne soit-elle, a sa psychopathologie: voir le livre de Freud ainsi intitulé (Psychopathologie de la vie quotidienne [1904]), l'un des premiers, en 1923, à avoir été traduits en français. Le premier chapitre s'ouvre sur... l'oubli concernant l'usage de noms propres et de langues étrangères, ot analyse le processus intervenu lors de l'oubli du nom d'un important... peintre (Signorelli). Et si Duchamp désignait cette ouverture en signant (sign/ Sign-) son nom sur telle petite feuille (slip / S-li), tout simplement, la condensation étant telle qu'elle s'étend jusqu'à « ce livre " que devient, en traduction, la Boifte Verte, non-livre s'il en est. Et s'il mettait en scène, par oubligeance envers cet ami (voir note 15) à l'occasion d'une oublication, “ la " formule du mécanisme, en la signant comme " on " signe un ready-made. L'oubli du nom de Signorelli est d'abord raconté dans une lettre (22 septembre 1898) à Fliess (parue dans La naissance de la psychanalyse [1950], Paris, PUF, 1956), puis dans un article écrit quelques jours plus tard ( $\propto$ Sur le mécanisme psychique de l'oubli » [1898], traduit dans Résultats, idées, problèmes, tome I, PUF, 1984) et dont la matière est résumée dans le premier chapitre en question.

34 Michel Sanouillet, lettre (17 octobre 1977) à André Gervais: * C'est ce jourlà, qu'entre autres jeux de mots, nous avions retenu la possibilité d'intituler ce nouveau recueil Duchamp du signe. Une autre suggestion (Prendre Duchamp) n'avait pas eu l'heur de lui plaire." Duchamp du signe (coiffant un regroupement bien incomplet paru en 1975), c'est à la fois, bien sûr, a du champ du signe " et " du chant du cygne". Voir note 25. 